\title{
Research Paper \\ The Effect of an 8-week Exercise Program Focused on Anticipatory Postural Ad- justments on Postural Control and Motor Function in the Elderly
}

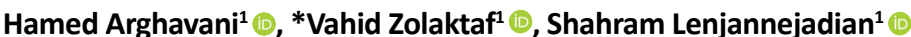

1. Department of Corrective Exercises, Faculty of Physical Education, University of Isfahan, Isfahan, Iran.

\begin{tabular}{|c|c|}
\hline $\begin{array}{l}\text { Use your device to san } \\
\text { and read the article online }\end{array}$ & \\
\hline 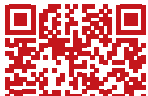 & $\begin{array}{l}\text { tory Postural Adjustments on Postural Control and Motor Function in the Elderly (Persian)]. Iranian Journal of Ageing. 2020; } \\
\text { 15(2):236-245. https://doi.org/10.32598/sija.2020.3.220 }\end{array}$ \\
\hline atresta & dol'https://doi.org/10.32598/sija.2020.3.220 \\
\hline
\end{tabular}

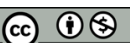

Received: 21 Jul 2019 Accepted: 23 Jul 2019 Available Online: 01 July 2020

Key words: Balance, Anticipatory postural adjustments Exercise, Elderly

\section{A B S T R ACT}

Objectives Improving balance and motor function in the elderly for preventing falling and its irreparable consequences has attracted the attention of many researchers in the field of aging. The purpose of the present is to investigate the effect of an exercise program based on Anticipatory Postural Adjustments (APAs) on postural control and motor function of the elderly.

Methods \& Materials In this experimental study conducted in 2017, the study population consists of 38 eligible male elderly people with a history of falls at least once in the last six months living in Isfahan, Iran in two groups: 18 in the test group (mean age $=70.4 \pm 3.2$, mean height $=65.3 \pm 6.2 \mathrm{~cm}$, mean weight= $65.3 \pm 4.2 \mathrm{~kg}$ ) and 15 in the control group (mean age $=69.6 \pm 3.1$ years, mean height $=167 \pm 5.5 \mathrm{~cm}$, mean weight $=67.3 \pm 6 \cdot 8)$. A foot-scan device was used to measure their postural control, and the Timed Up and Go (TUG) test was taken to measure their motor function. Data were analyzed in SPSS v. 20 software using repeated measures ANOVA at the significance level of $\mathrm{P} \leq 0.05$.

Results The exercise program significantly improved the mean postural control scores in anterior/posterior direction from $28.1 \pm 6.2$ to $20.5 \pm 2.4$ ( $F=8.21, P \leq 0.05)$, in medial/lateral direction from $17.1 \pm 1.5$ to 13.2 $\pm 2.9(F=13.4, P \leq 0.05)$, and in overall from $312.4 \pm 21.2$ to $285.8 \pm 17.5(F=10.02, P \leq 0.05)$; It also improved the mean TUG test score from $13.3 \pm 2.1$ to $10.1 \pm 1.0(F=16.02, P \leq 0.05)$. The experimental group showed a better performance than the control group after exercise in TUG test and postural control parameters $(\mathrm{P} \leq 0.05)$.

Conclusion An 8-week exercise program focused on APAs can be effective in improving postural control and motor function of the elderly.

\section{Extended Abstract}

\section{Introduction}

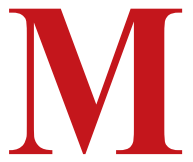

any researchers believe that loss of postural balance in the face of perturbation is the main reason for the fall in the elderly people [1]. The central nervous system uses two anticipatory and compensatory postural adjustments to maintain, restore, and control postural balance under perturbation. If the anticipatory mechanism be activated in time, earlier or with less delay, the need for more activity of the compensating mechanism will be reduced and, therefore, the displacement of the center of gravity will decrease and the postural balance will be maintained better or restored sooner $[2,3]$.

\section{* Corresponding Author:}

Vahid Zolaktaf, PhD.

Address: Department of Corrective Exercises, Faculty of Physical Education, University of Isfahan, Isfahan, Iran.

Tel: +98 (913) 1130369

E-mail: v.zolaktaf@spr.ui.ac.ir 
Postural control mechanisms in the elderly are impaired [4]. Recently, researchers have proven the possibility of improving the anticipatory mechanism of postural balance control in the elderly with exercises for receiving and throwing the ball [5]. Due to the novelty of these exercises and the lack of a specific training protocol in this area, the aim of this study was to evaluate the effect of an eight-week training protocol focused on the Anticipatory Postural Adjustments (APAs) on the postural control and motor function of the elderly people.

\section{Methods \& Materials}

In this experimental study conducted in 2017, the study population consists of male elderly people in Isfahan, Iran with a history of falls at least once in the last six months. Considering $\alpha=0.05, \beta=0.8$ and effect size $=0.3$ in G*POWER software, 15 samples for each group were calculated, which due to the possibility of sample drop, it was determined 20. After considering the inclusion criteria, 40 eligible individuals were selected and randomly divided into two equal groups. Of them, 18 in the test group (mean age $=70.4 \pm 3.2$, mean height $=165.3 \pm 6.2 \mathrm{~cm}$, mean weight $=65.3 \pm 4.2 \mathrm{~kg}$ ) and 15 in the control group (mean age $=69.6 \pm 3.1$ years, mean height $=167 \pm 5.5 \mathrm{~cm}$, mean weight $=67.3 \pm 6.8$ ) completed the evaluations.

The experimental group participated in the exercise program for 8 weeks, 3 sessions per week each for 1 hour. The main movements were a set of throwing and receiving exercises, which included the variables of throwing distance (far or near), throwing direction (high up in the air or down to the ground), throwing type (overhead or lower to chest), weight of the ball (light or heavy), type of the ball (basketball, volleyball, medical), posture (increased or reduced base of support), sitting position on a chair (fixed or adjustable) were manipulated during the program. Footscan 7 (RSscan International, Olen, Belgium) was used to measure postural control of the elderly.

After determining the dominant leg, the subjects were asked to stand on the device with the dominant leg barefoot and with eyes open. The duration of the test was three repetitions of 30 seconds with two minutes of rest between each attempt. The average of these repetitions was recorded as an individual record for further calculations. The Timed Up and Go (TUG) test was used to measure functional mobility of the subjects. It uses the time when the subject rises from a chair with no arms, walks three meters with maximum speed, and then walks back to the chair and sits down. Each subject performed the test twice and the best recorded time was considered as his record. After eight weeks of exercise, the pre-tests were performed again. Repeated-measures ANOVA was used for the analysis of time and group effects at a significance level of 0.05 .

\section{Results}

According to Table 1, the results of within-group and between-group comparisons showed that the eight-week exercise program improved anterior/posterior control, medial/ lateral control, overall postural control, and TUG test score

Table 1. Repeated-measures ANOVA results

\begin{tabular}{|c|c|c|c|c|c|c|}
\hline \multirow{2}{*}{ Variables } & \multirow{2}{*}{ Group } & \multicolumn{2}{|c|}{ Mean \pm SD } & \multirow{2}{*}{$\begin{array}{l}\text { Within-group } \\
\text { Comparison }\end{array}$} & \multirow{2}{*}{$\begin{array}{l}\text { Between-group } \\
\text { Comparison }\end{array}$} & \multirow{2}{*}{$\begin{array}{l}\text { Interaction } \\
\text { Effect }\end{array}$} \\
\hline & & Pre-test & Post-test & & & \\
\hline \multirow{2}{*}{$\begin{array}{l}\text { Anterior/posterior } \\
\text { control }(\mathrm{cm})\end{array}$} & Test $(n=18)$ & $28.02 \pm 6.22$ & $20.52 \pm 2.41$ & $\begin{array}{c}\mathrm{F}=8.21 \\
\mathrm{P}=0.012^{*}\end{array}$ & \multirow{2}{*}{$\begin{array}{c}F=5.88 \\
P=0.031^{*}\end{array}$} & \multirow{2}{*}{$\begin{array}{c}\mathrm{F}=14.33 \\
\mathrm{P}=0.002^{*}\end{array}$} \\
\hline & Control (n=15) & $29.53 \pm 5.52$ & $27.13 \pm 4.22$ & $\begin{array}{l}F=1.54 \\
P=0.213\end{array}$ & & \\
\hline \multirow{2}{*}{$\begin{array}{l}\text { Medial/lateral } \\
\text { control }(\mathrm{cm})\end{array}$} & Test $(n=18)$ & $17.09 \pm 5.12$ & $13.20 \pm 2.90$ & $\begin{array}{c}F=13.45 \\
P=0.019^{*}\end{array}$ & \multirow{2}{*}{$\begin{array}{c}F=7.49 \\
P=0.002^{*}\end{array}$} & \multirow{2}{*}{$\begin{array}{l}F=17.89 \\
P=0.019^{*}\end{array}$} \\
\hline & Control (n=15) & $19.78 \pm 4.55$ & $19.40 \pm 19.40$ & $\begin{array}{l}F=2.08 \\
P=0.302\end{array}$ & & \\
\hline \multirow{2}{*}{$\begin{array}{l}\text { Overall postural } \\
\text { control }(\mathrm{cm})\end{array}$} & Test $(\mathrm{n}=18)$ & $312.41 \pm 21.20$ & $285.79 \pm 17.51$ & $\begin{array}{c}\mathrm{F}=10.2 \\
\mathrm{P}=0.001^{*}\end{array}$ & \multirow{2}{*}{$\begin{array}{c}F=5.38 \\
P=0.022^{*}\end{array}$} & \multirow{2}{*}{$\begin{array}{c}F=6.91 \\
P=0.001 *\end{array}$} \\
\hline & Control (n=15) & $301.14 \pm 22.85$ & $309.89 \pm 23.60$ & $\begin{array}{l}F=1.95 \\
P=0.237\end{array}$ & & \\
\hline \multirow{2}{*}{ TUG test score } & Test $(n=18)$ & $13.31 \pm 2.11$ & $10.06 \pm 10.06$ & $\begin{array}{c}\mathrm{F}=16.2 \\
\mathrm{P}=0.001^{*}\end{array}$ & \multirow{2}{*}{$\begin{array}{l}F=12.65 \\
P=0.018^{*}\end{array}$} & \multirow{2}{*}{$\begin{array}{l}\mathrm{F}=12.11 \\
\mathrm{P}=0.036^{*}\end{array}$} \\
\hline & Control ( $n=15)$ & $14.83 \pm 2.03$ & $14.58 \pm 2.45$ & $\begin{array}{l}F=3.11 \\
P=0.114\end{array}$ & & \\
\hline
\end{tabular}

* There is a significant difference at $\mathrm{P}<0.05$. 
( $\mathrm{P} \leq 0.05)$; but in the control group, there were no significant changes $(\mathrm{P} \geq 0.05)$.

\section{Conclusion}

With the onset of aging, there is a decrease in motor function and balance. One of the reasons for this decrease is the reduced use of APAs in the elderly. Therefore, APAs is significantly delayed in healthy elderly compared to young people, and the muscles with an activity similar to that of primary motor cortex muscles or the moment of impact are used [6]. Delayed muscle activity in the APAs phase in the elderly causes greater displacement of the center of mass after perturbation, indicating greater postural instability [7]. However, clinical practice has not specifically focused on improving the APAs. This may be due to the lack of evidence on the role of exercises focused on APAs in improving postural stability and motor fucntion in the elderly [5].

There, we suggest that APA-based exercises can improve the balance of the elderly and reduce falls in them. In all three postural control indices (anterior/posterior, medial/lateral and overall), a decrease in center of pressure displacement was observed after exercise, indicating an improvement the static balance. Moreover, the functional mobility of the elderly under TUG test decreased by 3.25 seconds, in average, after exercise. The TUG test score is related to Berg Balance Scale score and walking speed; hence, the improvement in the score of TUG test in our study shows that the postural stability of the elderly was improved and they were less likely to be at risk of falling.

It seems that an 8-week training intervention focused on APAs which included throwing and receiving a ball with different weights and sizes and in different directions and positions, can improve postural stability in the elderly. It is possible that a APA-based training program reduces the need to activate the compensatory mechanism by timely activation of the muscles involved in APAs, and reduces the displacement of the center of gravity. These exercises are an effective way to improve the APAs and, thus, postural balance and motor function in the elderly which eventually prevents falling and its irreparable consequences.

Corrective exercise specialists and physiotherapists who work with older adults can use this program along with other training protocols to improve balance and motor function of the older people. The limitations of the present study include: the statistical population limited to one city, short study duration, uncontrolled mental and psychological conditions of the subjects, the use of a convenience sampling technique, and the effect of individual and hereditary differences on test results.

\section{Ethical Considerations}

\section{Compliance with ethical guidelines}

This study was approved by the Ethics Committee of University of Isfahan (Code: IR.UI.REC1396, 065). All the procedures of the study were explained to participants, and all their questions were answered before they were asked to participate in the study.

\section{Funding}

The present paper was extracted from the $\mathrm{PhD}$. thesis of the first author, Department of Corrective Exercises, Faculty of Physical Education, University of Isfahan, Isfahan.

\section{Authors' contributions}

All authors equally contributed in preparing this article.

\section{Conflicts of interest}

The authors declared no conflict of interest

\section{Acknowledgements}

We wish to express our sincere gratitude to all the elderly, and the University of Isfahan whose cooperation has been a great source of help. 


\title{
تأثير هشت هفته تمرينات متمركز بر مكانيسم ييشبين كنترل تعادل بر كثترل قامت و عملكرد حركتى سالمندان
}

حامد ارغوانى' '•، •وحيد ذوالاكتاف' نه، شهرام لنجان نزاديان'

ا.كروه تمريثات اصلاحى، دانشكده تربيت بدنى، دانشكاه اصفهان، اصفهان، ايران.

\begin{abstract}
בתו

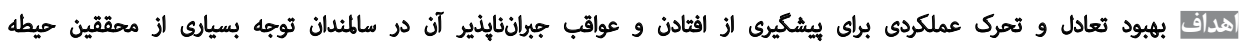

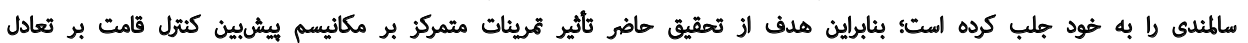
وعملكرد هركثي سالمثندان بود.

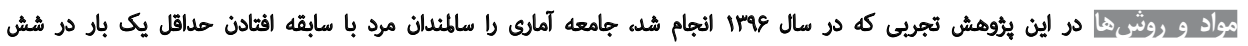

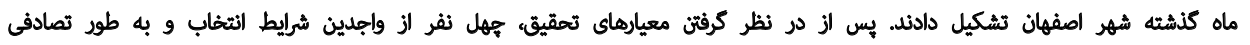

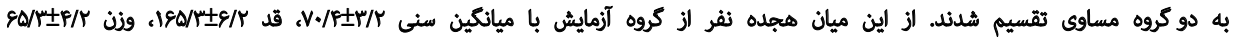

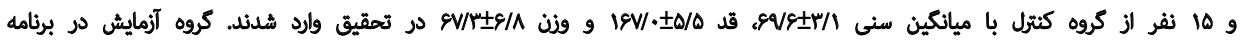

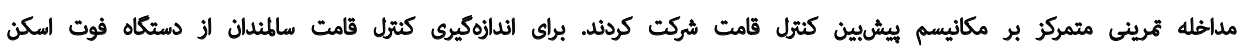

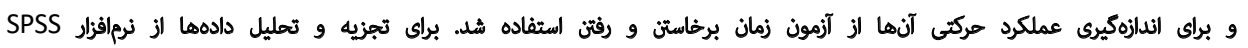

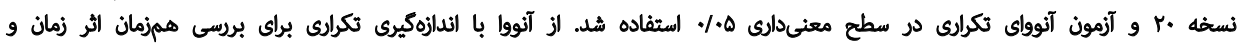

اثركروه استفاده شد.

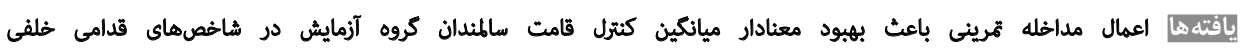

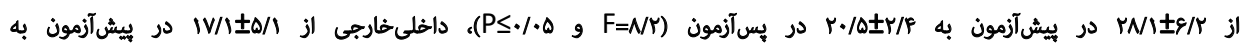

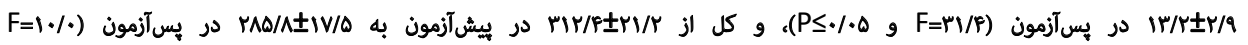
و

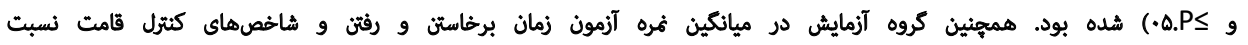

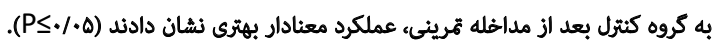

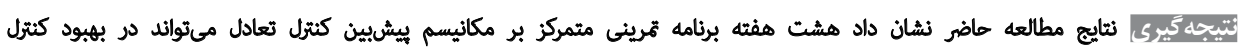

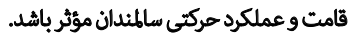

تاريخ دريافت: •r تير 199

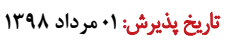

تاريخ انتشار: 11 تير 1499

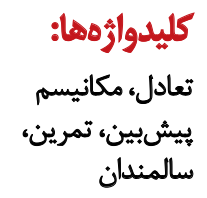

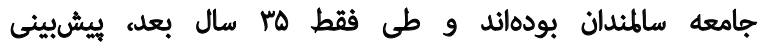

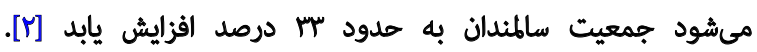

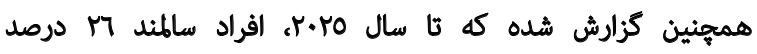

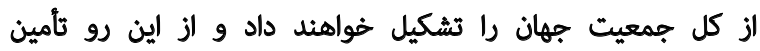

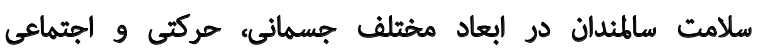

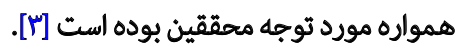

افزايش سن به واسطه اغتلال در عملكرد حركتى، با كاهش توانايى نكهارى قامت بدن و افت عملكرد سيستمهاى كنترل

سالمندى بخشى الز فرايند زيستى الست كه ثقام موجودات

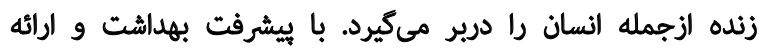

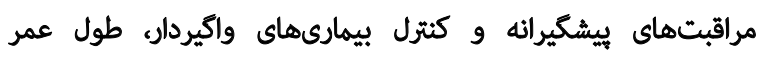

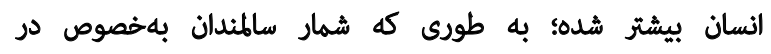

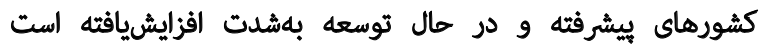

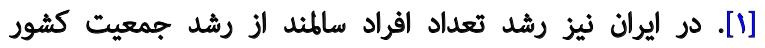

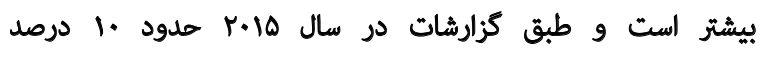

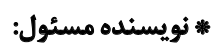

وحيد ذوالاكثاف

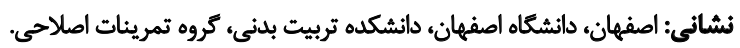
تلفن: v.zolaktaf@spr.ui.ac.ir يست الكترونيكي 
است [•1]. همجنين هئل تهقيق ديكر نشان داده است كه

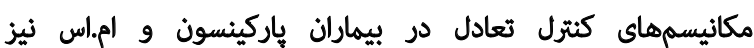

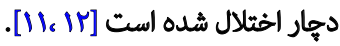

در تحقيقى ديكر از تمرينات يكجلسهاي، شامل يرتاب و دريافت

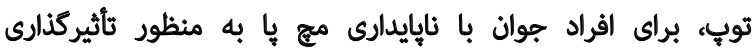

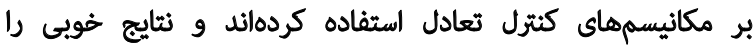

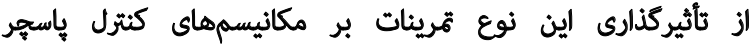

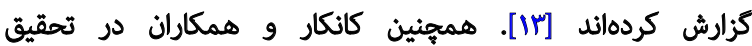

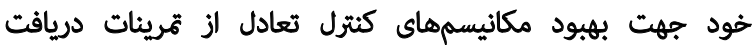

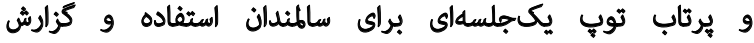

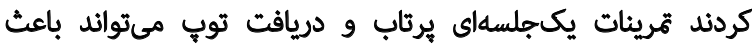

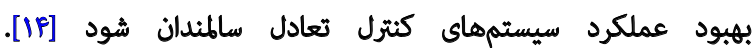
در ادامه محققين به معرفى يك روش ابداعى جديد براي بهبود فئرد

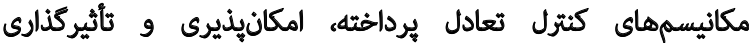

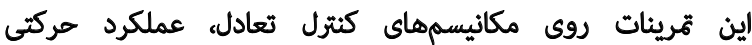

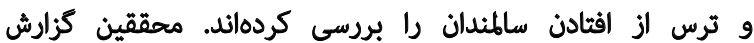

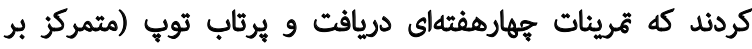

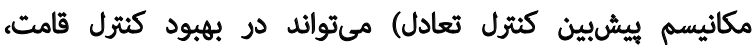

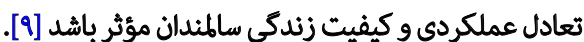
بنابراين با توجه به جديد بودن اين حيطه از تمرينات، ناشناخته

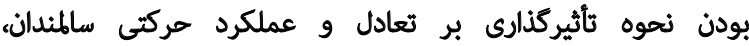

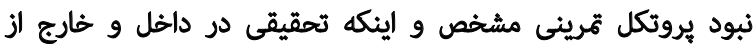
كشور در اين زمينه كه از مترينات بلندمدت متمركز بر بر مكانيسم

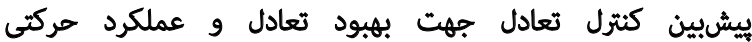

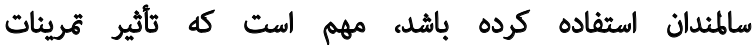

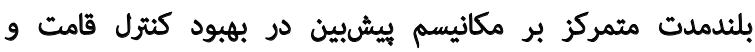
تصرك عملكردى بيشتّ مطالعه شود.

dillbo

تحقيق حاضر الز نوع تجربى با طرح ييشآزمون يسآزمون و

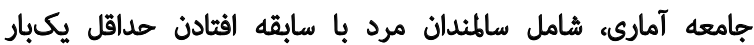

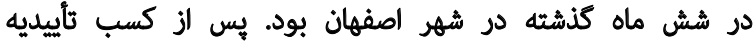

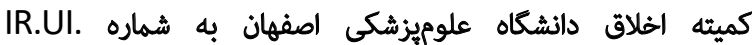
REC1396,065 و هماهنكى با مراكز و نهادهاى مريوطه و و بانئ مراجعه به ياركها و مساجد و توزيع بركهاى شركت داوطلبانه

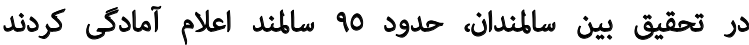

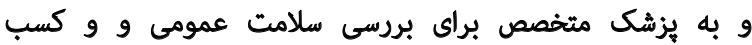

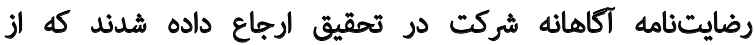

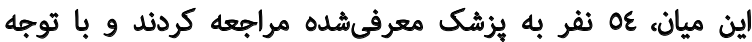

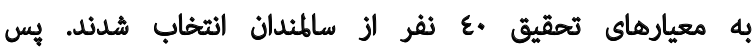

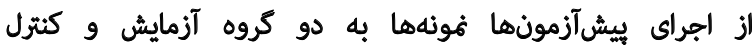

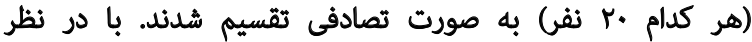

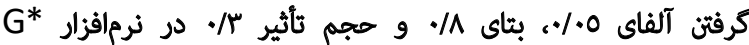

قامت همراه است كه خود باعث كاهش تهرى عملكردى 9

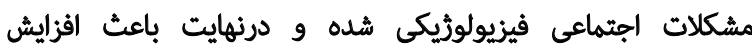

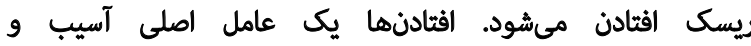

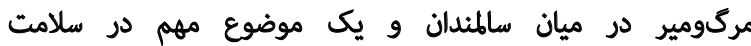

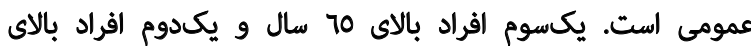

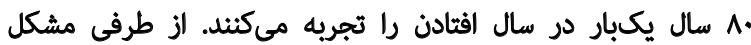

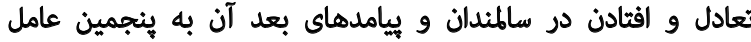

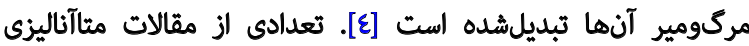

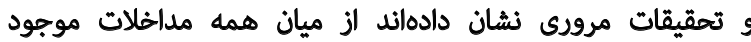

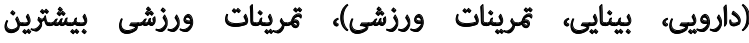

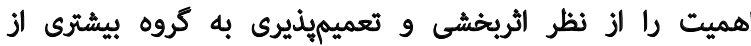

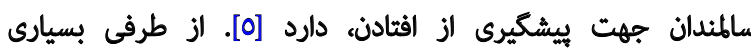

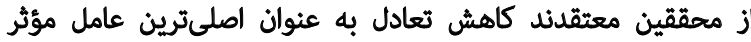

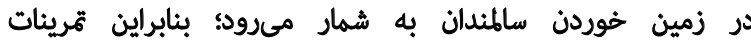

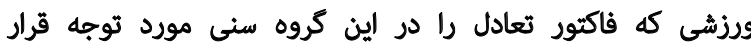

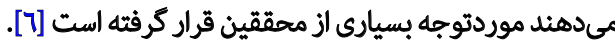

سيستم عصبى مركزى از دو مكانيسم كثترلى مختلف براي

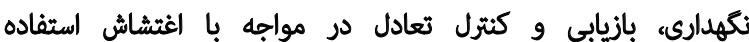
مىكند. مكانيسم اول شامل كنترل تعادل فيد فوروارد يا تنظيمات

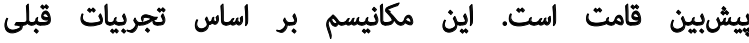

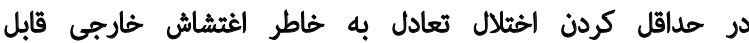

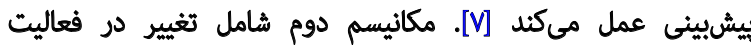

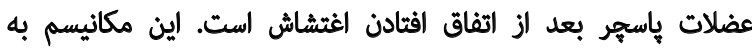

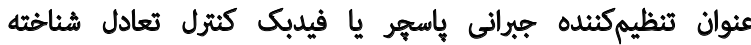

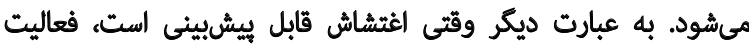

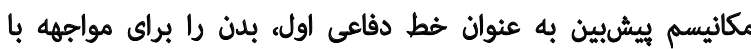

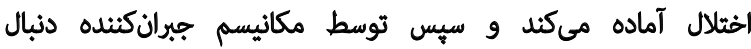

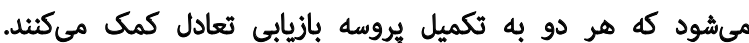

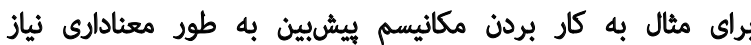

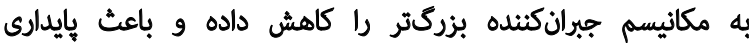

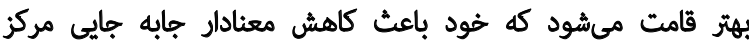

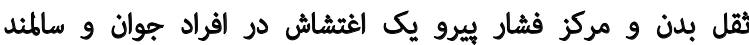

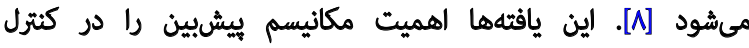

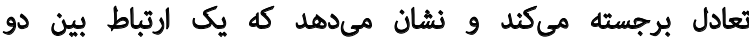

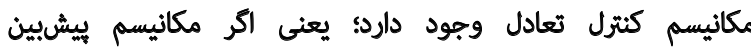

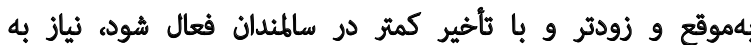
جابهجايى بيشتر مركز ثقل كاهشيافته و الز اين طريق تعادئ دادل بيشتر

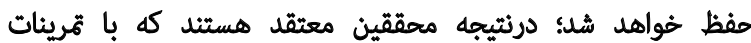

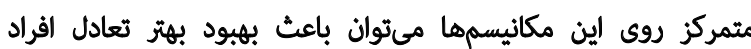
سالمند و كاهش افتادن در سالمئدان شيد [9]].

مشخصشلهد مكانيسمهاى كنتزل تعادل در افراد داراى مشكل

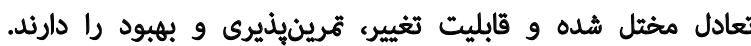

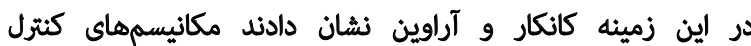
تعادل در افراد سالمند نسبت به افراد جوان دهار اختلال شده 


\begin{tabular}{|c|c|c|c|c|c|}
\hline \multicolumn{4}{|c|}{ Time (ms) } & \multicolumn{2}{|c|}{4360} \\
\hline \multicolumn{4}{|c|}{ Selection } & \multicolumn{2}{|c|}{19.980 seconds } \\
\hline \multicolumn{4}{|c|}{ Total Time } & \multicolumn{2}{|c|}{19.980 seconds } \\
\hline \multicolumn{4}{|c|}{ Recording speed } & \multicolumn{2}{|c|}{50 frames/sec } \\
\hline \multicolumn{4}{|c|}{ Total number of frames } & \multicolumn{2}{|c|}{999 \#Frames } \\
\hline & \multicolumn{2}{|c|}{ Minimum } & \multicolumn{2}{|c|}{ Maximum } & Delta \\
\hline $\operatorname{COF} x$ & \multicolumn{2}{|c|}{$269 \mathrm{~mm}$} & \multicolumn{2}{|c|}{$274 \mathrm{~mm}$} & $5 \mathrm{~mm}$ \\
\hline COFY & \multicolumn{2}{|c|}{$121 \mathrm{~mm}$} & & $5 \mathrm{~mm}$ & $15 \mathrm{~mm}$ \\
\hline \multicolumn{4}{|c|}{ COF Total traveled way } & \multicolumn{2}{|c|}{$205 \mathrm{~mm}$} \\
\hline & & \multicolumn{3}{|c|}{$\operatorname{COF} \mathrm{X}$} & COF $Y$ \\
\hline \multicolumn{2}{|c|}{ Current COF (mm) } & \multicolumn{3}{|c|}{272} & 127 \\
\hline
\end{tabular}

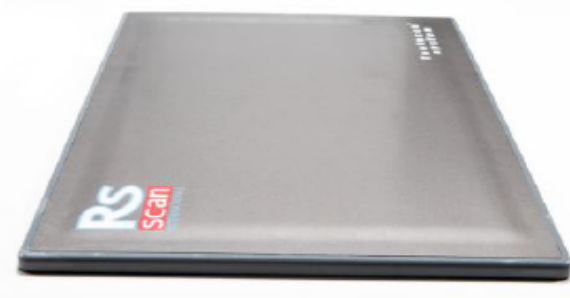

L

تصوير ا. دستكاه فوت اسكن وخروجى ميانكين جابهجايى فشار در شاخصهاي قدامى خلفى، داخلى خارجى وكل

تكرار •"ثانيهاي همراه با ب دقيقه استراهت در بين هر تلاش

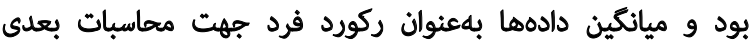
ثبت شد.

براى الندازهكيرى تحرى عملكردى سالمندان الز آزمون زمان

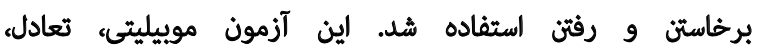

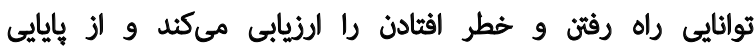

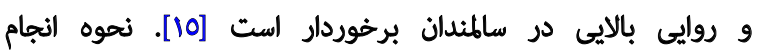

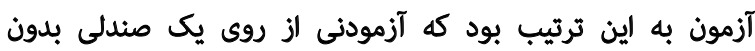

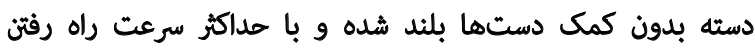

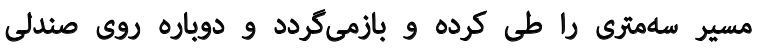

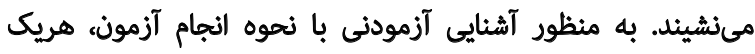

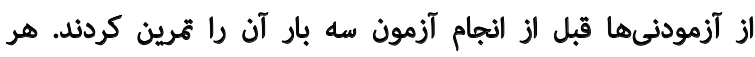

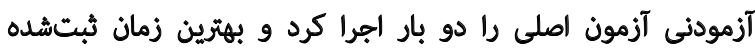

بهنوان ركورد وي ثبت شدئ أروني

يس از اجراى هشت هفته قمرين، ييشآزمونها مجدد اجرا

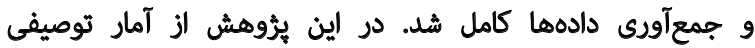

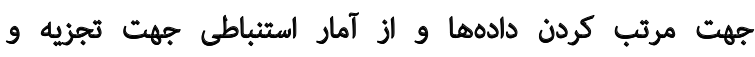

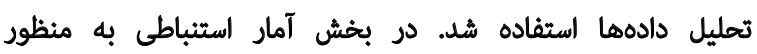

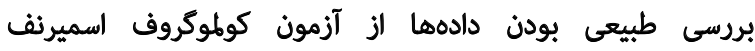

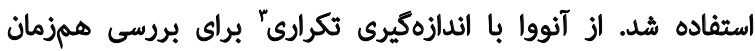

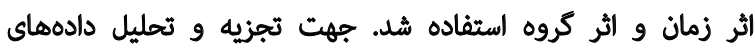

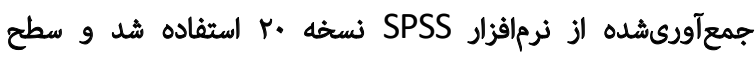

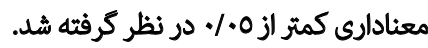

batil

هدول شماره 1 ويزٔكىهاي جمعيتشناختى آزمودنىهاى اين

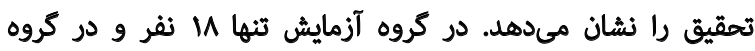
كنتزل 10 نفر موفق به تكميل مراحل اندازهيرى شدئد.

با توجه به جدول شماره ا، بين كروهها الز نظر ويرٔكىهاى
نسخه POWER

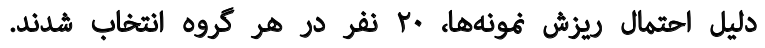

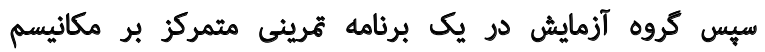

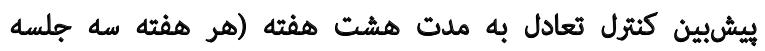

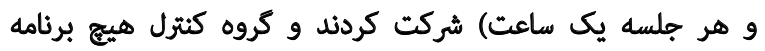

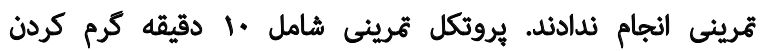

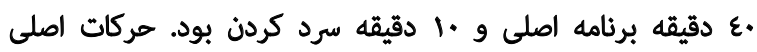

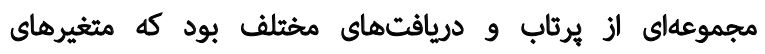

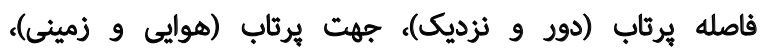

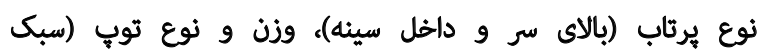

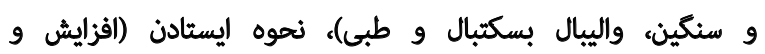

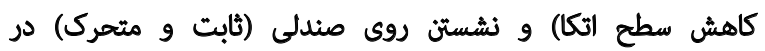
طول برنامه دست كاري شدند.

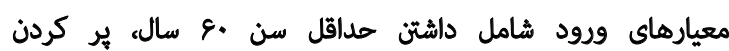

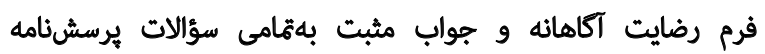

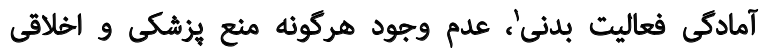

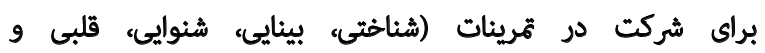

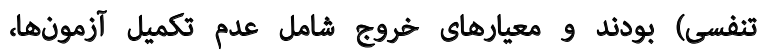
داشتن ناهنجارىهاى كف يا و عدم شركت در تمرينات بود.

براي اندازهيرى كنتزل قامت سالمندان الز فوت اسكنr ساخت

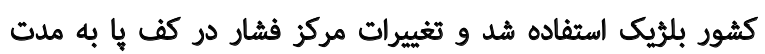

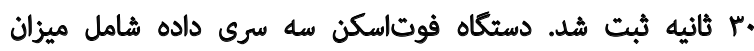

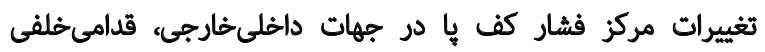

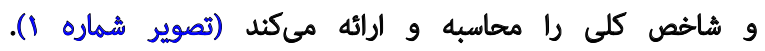
ابتدا فرد با لباس راحت به به مدت هينج دقيقه كرم كردن شامل

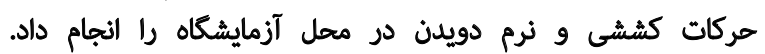

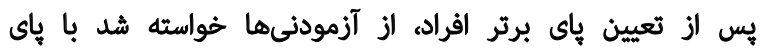

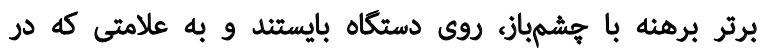

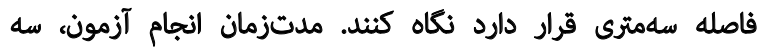


جدول ا. مشخصات جمعيتشناختى آزمودنى ها به تفكيك تروههاى تحقيق

\begin{tabular}{|c|c|c|c|c|}
\hline \multicolumn{3}{|c|}{ ميانكين ثانحرافمعيار } & \multirow{2}{*}{ تعداد آزمودنى } & \multirow{2}{*}{ وضعيت } \\
\hline سن (سال) & وزن (كيلوكئرم) & قد (سائتى متر) & & \\
\hline$V \cdot(f \pm r / r)$ & $\varepsilon \Delta / T \pm Y / T r$ & IEA/TIF/Te & M & كروه آزمايش \\
\hline$e q / F \pm r / . q$ & 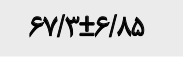 & $\mid e V / \cdot \pm \Delta / \propto q$ & 10 & كروه كتترل \\
\hline$+\mid A+1$ & .1889 & driv & - & مقدار احتمال \\
\hline
\end{tabular}

\begin{tabular}{|c|c|c|c|c|c|c|}
\hline \multirow{3}{*}{ اثر مثقابل } & \multirow{3}{*}{ مثايسه بين كروهى } & \multirow{3}{*}{ مقايسه درون كروهي } & \multicolumn{4}{|c|}{ جدول لا. نتايج آزمون آنووا با اندازههاى تكرارى براى متغيرهاى اندازهكيرى تحقيق } \\
\hline & & & \multicolumn{2}{|c|}{ ميانكين+انحرافمعيار } & \multirow{2}{*}{ 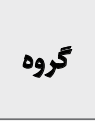 } & \multirow{2}{*}{ مثغيرها } \\
\hline & & & 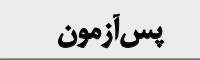 & 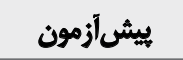 & & \\
\hline \multirow{2}{*}{$\begin{array}{l}\mathrm{F}=r^{e} / / \mathrm{m} \\
\mathrm{P}=* / * \cdot r^{*}\end{array}$} & $F=\Delta / M$ & $\begin{array}{l}\mathrm{F}=N T \mid \\
\mathrm{P}=*|\cdot| T^{*}\end{array}$ & $r \cdot / \Delta T \pm T / F I$ & $r N \cdot r \pm E / T r$ & $\begin{array}{l}\text { آزمايش } \\
\text { n=|1 }\end{array}$ & \multirow{2}{*}{ قدامى خلفى(cm) } \\
\hline & $\mathrm{P}=\cdot \mid \cdot \mathrm{r})^{*}$ & $\begin{array}{l}\mathrm{F}=1 / \Delta)^{\circ} \\
\mathrm{P}=. / T / T\end{array}$ & $r / N \Psi+T / R r$ & $r q / \Delta \pm \Delta r / \Delta r$ & $\underset{n=10}{S}$ & \\
\hline \multirow{2}{*}{$\begin{array}{l}F=1 Y / 19 \\
P=.1 .19^{*}\end{array}$} & \multirow{2}{*}{$\begin{array}{l}F=V / F q \\
P=* / . . r^{*}\end{array}$} & $\begin{array}{l}F=1 \% / F 0 \\
P=.1 .19^{\circ}\end{array}$ & $1 r / r+ \pm r / 9$. & $I V / \Delta \pm+9 / T$ & $\begin{array}{l}\text { آزمايش } \\
\text { n=|1 }\end{array}$ & \multirow{2}{*}{ داخلى خارجى(cm) } \\
\hline & & $\begin{array}{l}F=r / \cdot A \\
P=* / r \cdot r\end{array}$ & $19 / 6 \cdot \pm r / 8 A$ & $19 / \digamma \pm Y N \Delta \Delta$ & كثترل & \\
\hline \multirow{2}{*}{$\begin{array}{l}\mathrm{F}=8 / 91 \\
\mathrm{P}={ }(\cdots)^{*}\end{array}$} & \multirow{2}{*}{$\begin{array}{c}F=\Delta / r \Lambda \\
P=. / \cdot r T^{*}\end{array}$} & $\begin{array}{l}\mathrm{F}=1+/ \mathrm{r} \\
\mathrm{P}=+1++1^{*}\end{array}$ & $r \wedge \Delta / V q \pm|V / \Delta|$ & $M|r / r| \pm F I / r$. & $\begin{array}{l}\text { آزمايش } \\
n=\mid \lambda\end{array}$ & \multirow{2}{*}{ شاخص كل(cm) } \\
\hline & & $\begin{array}{l}F=1 / 9 \Delta \\
P=. / \pi T V\end{array}$ & $r \cdot q / A q \pm \pi / 8$. & $\Gamma \cdot 1 / T Y \pm 1 Y / \Lambda \Delta$ & كنترل & \\
\hline \multirow{2}{*}{$\begin{array}{l}\mathrm{F}=|r / M| \\
\mathrm{P}=+/+r \xi^{*}\end{array}$} & $F=\mid r / \& \Delta$ & $\begin{array}{l}F=18 / \pi \\
P=.1 \cdot+1^{*}\end{array}$ & $1.1 .8 \pm 1 / .$. & $|r / r \pm r| m \mid$ & 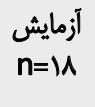 & \multirow{2}{*}{ زمان برخاستن و } \\
\hline & $\mathrm{P}=+1+1 \mathrm{~N}^{*}$ & $\begin{array}{c}F=m / M \\
P=* / M I f\end{array}$ & $I F / \Delta A \pm T / F \Delta$ & $\mid \varphi / r \pm \lambda \mu / \cdot r$ & كتترل & \\
\hline
\end{tabular}

次

كه هشت هفته تمرين در كروه آزمايش باعث بهبود معنادار

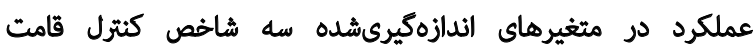

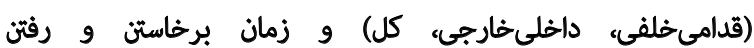

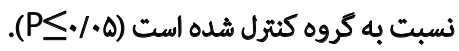

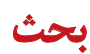
هدف الز تحقيق حاضر بررسى ثأثير تمرينات متمركز بر مكانيسم

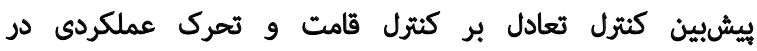

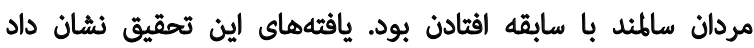

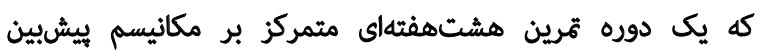

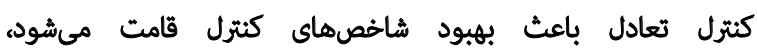
به اين معنى كه در هر سه شاخص كثترل قامت (قدامىخلفى،

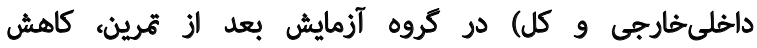

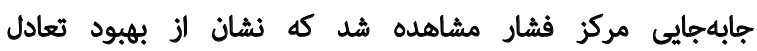

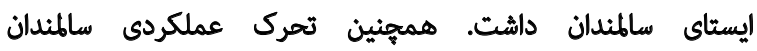

$$
\text { علامت" معنى دارى آناليز آمارى رانشان مى دهد. }
$$

جمعيتشناختى تفاوت معناداري وجود نداشته و اين ويخكىها

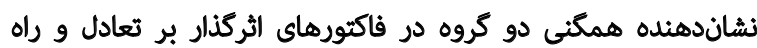

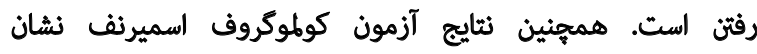

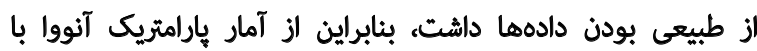
اندازهكيرى تكرارى استفاده شد. در جدول شماره r اطلاعات مربوط به آزمون آنووا با اندازهكيرى

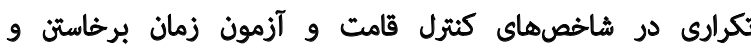
رفتي در دو كروه الرائه شده السته با توجه به جدول شماره r نتايج مقايسه درونكروهى نشان

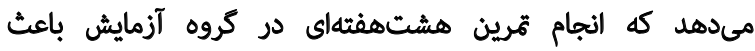

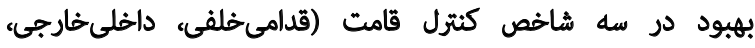

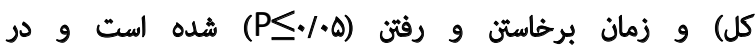

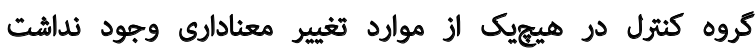

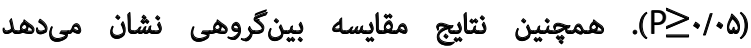


اين امر مىتواند ناشى الز فقدان شواهدى در مورد نقش تمرينات

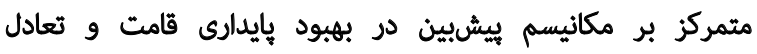

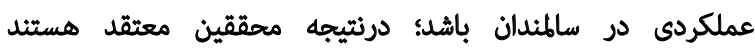

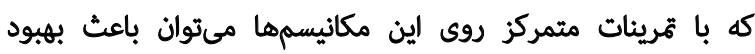

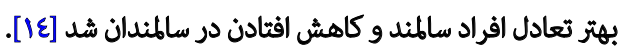

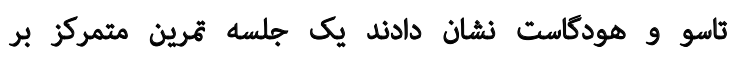

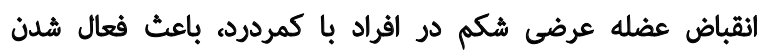

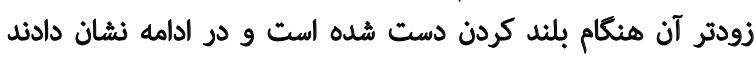

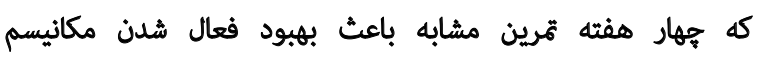

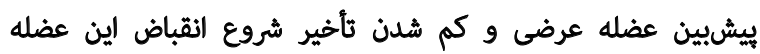

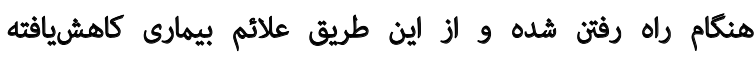

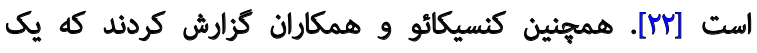

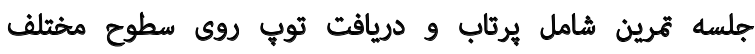

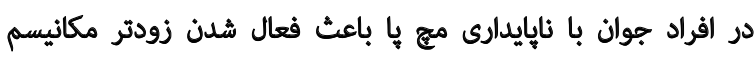

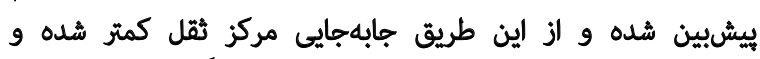

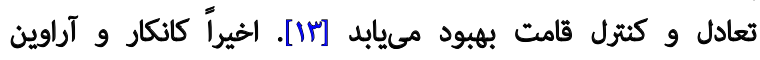

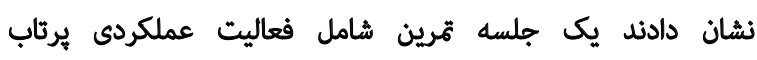

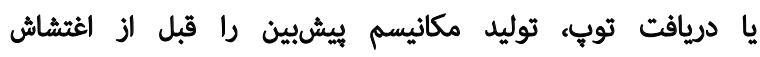

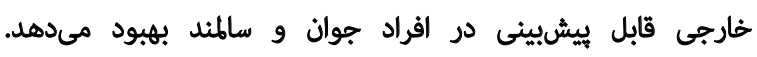

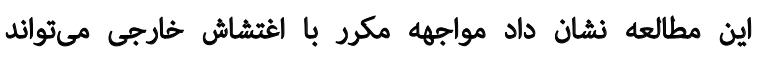

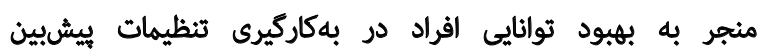

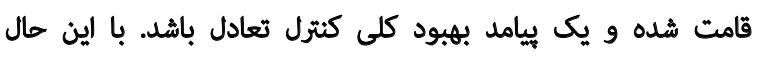

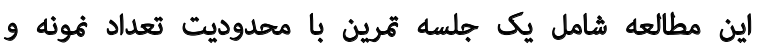

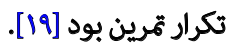

در ادامه جاك هان و همكاران كزارش كردهاند كه هيهار هفته

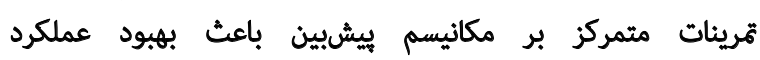

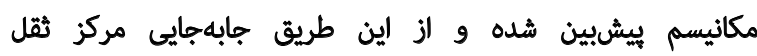

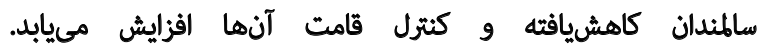

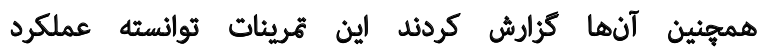

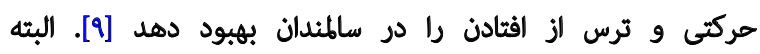

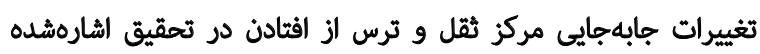

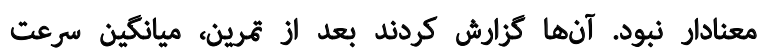

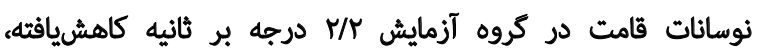

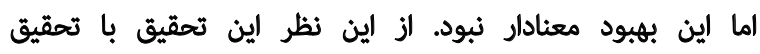

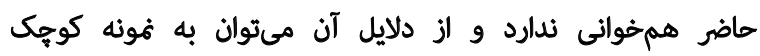

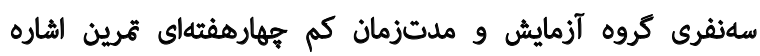

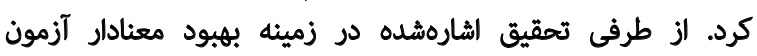

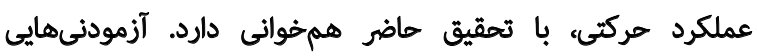

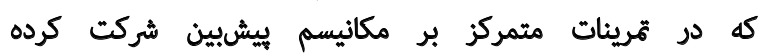

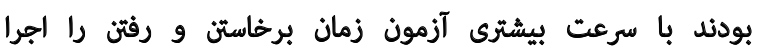

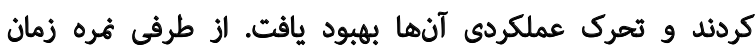

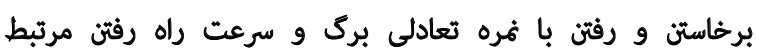

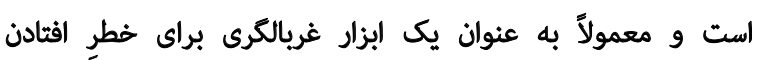

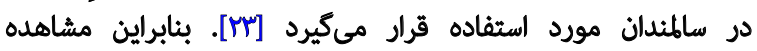

كروه آزمايش كه با آزمون زمان برخاستن و رفتن اندازهكيرى

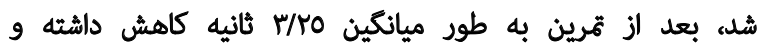

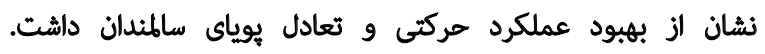

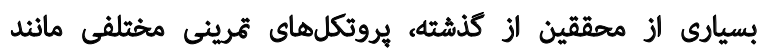

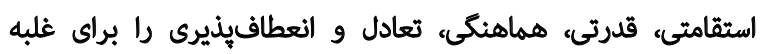

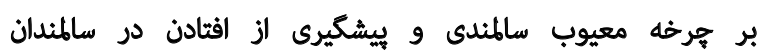

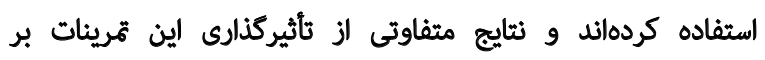

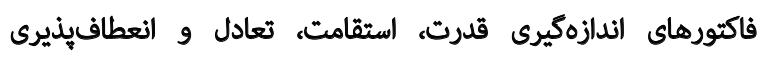

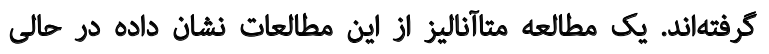

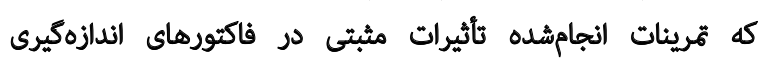

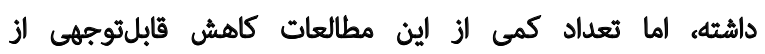

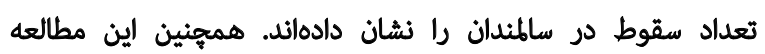

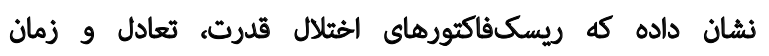

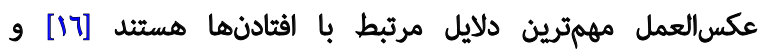

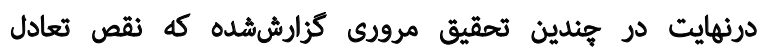

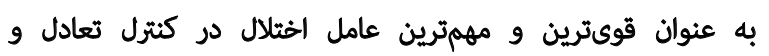

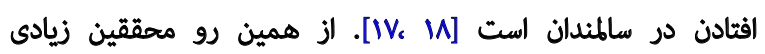

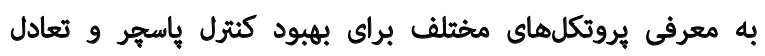

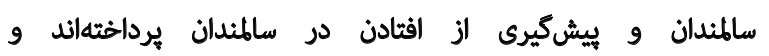

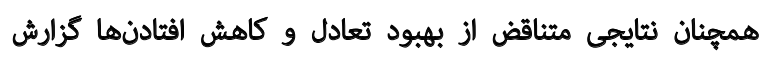

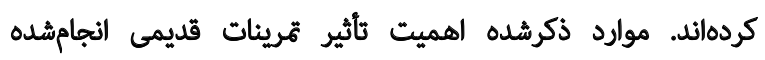

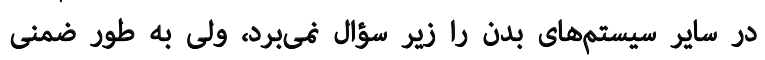

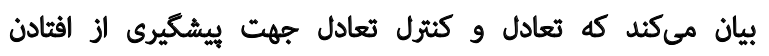

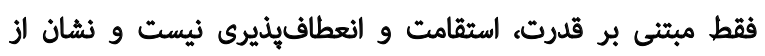

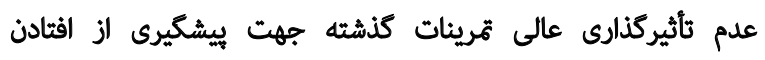

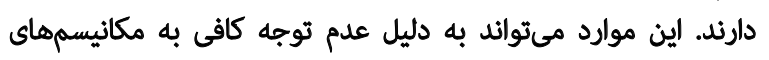

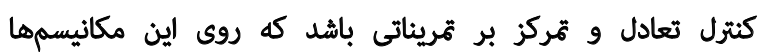
بيشترين تأثيركذارند [19]

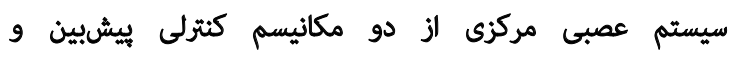

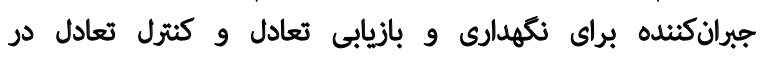

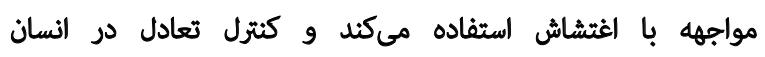

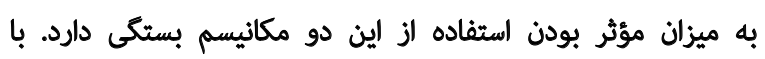

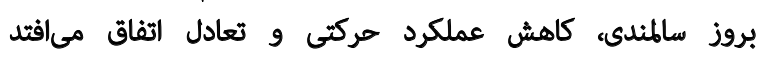

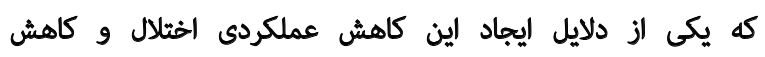

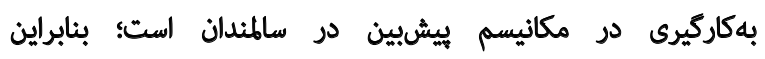

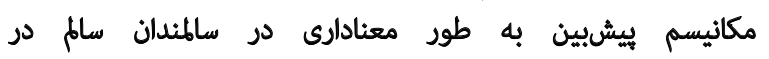

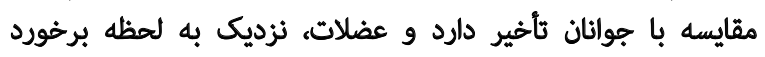

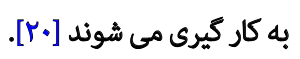

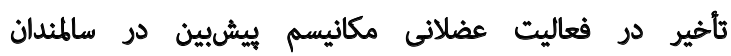

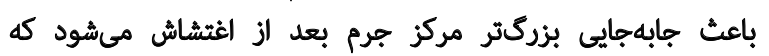

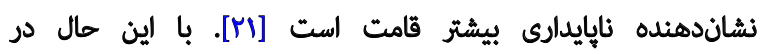

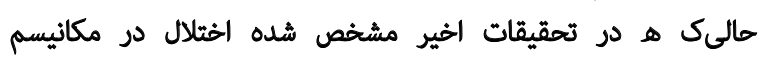

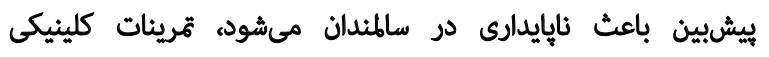
به طور ويزٔه بر بهبود مكانيسم ييشبين متمركز نشده است كه كاينان 


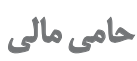

اين مقاله بركرفته از رساله دكترى حامد ارغوانى در كروه

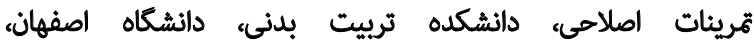

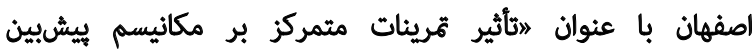

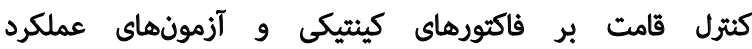
هركتى سالمندان در مواجهه با اغتشاش خارجىه (ست. مشاركت نويسند مثان

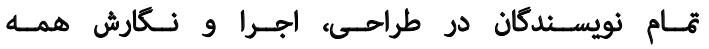

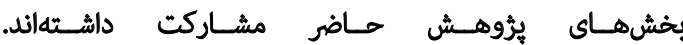

$$
\text { تعارض منافع }
$$

بنابر اظهار نويسندكان، اين مقاله تعارض منافع ندارد.

$$
\text { تشكر وقدودائي }
$$

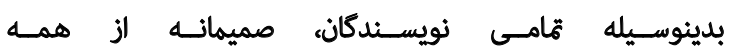

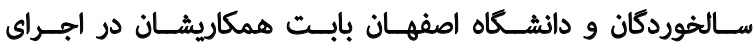
ايسن ئروهـش تشـكر و قدردانسى مىكنيـم.
بهببود ناشى الز تمرين در نهره اين آزمون نشان مىدهد در كروه

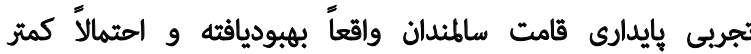

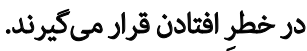

\section{نتيجلكيرىنهايي}

با توجه به ياقتهاي تحقيق حاضر ميتوان نتيجه كرفت

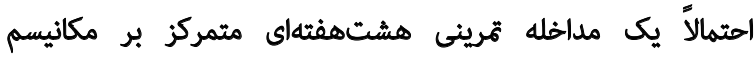

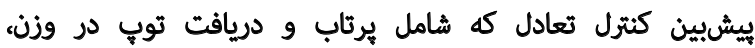

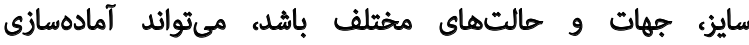

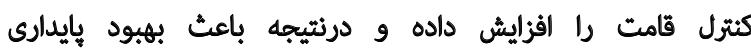

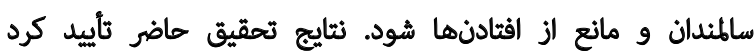

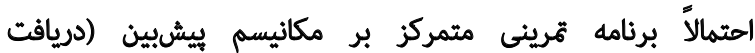

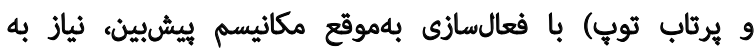

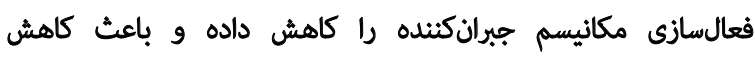

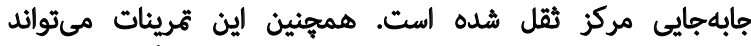

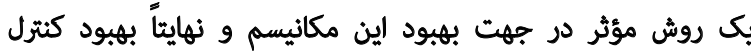

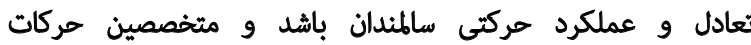

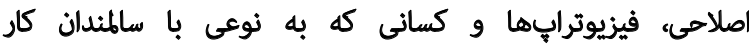

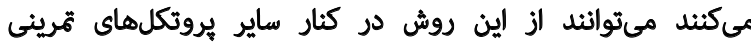

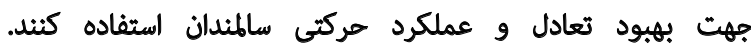

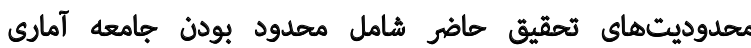
به شهر اصفهان، محدود بودن زمان النجام مطالعه، كنترل نشدن

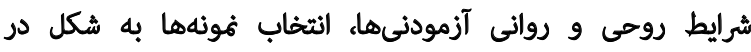

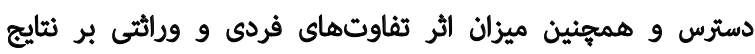

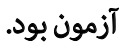

بيشنهاد مىشود تهقيقات ديكر با اندازهيرى الكترومايوكرافى ئى

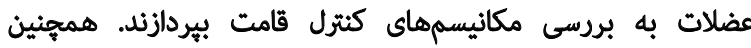

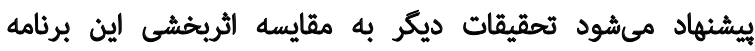

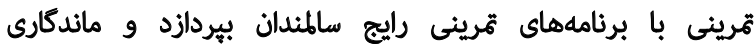

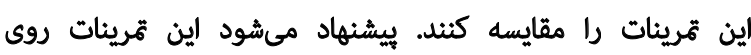
افرادى كه دهار نقص تعادل هستند الجرا و تأثيركذارى ثمرينات

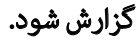

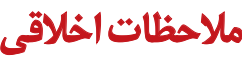

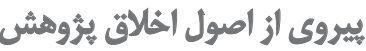

اين مطالعه توسط كميته اخلاق دانشكاه اصفهان تأييد شده

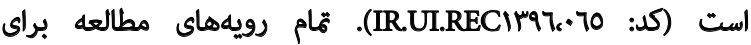

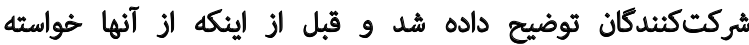

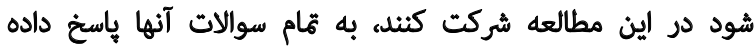




\section{References}

[1] Sattin R. Falls among older persons: A public health perspective. Annual Review of Public Health. 1992; 4(3):489-508. [DOI:10.1146/annurev.pu.13.050192.002421]

[2] Organization World Health. World report on ageing and health. Geneva: World Health Organization; 2015. https://www.who. int/ageing/publications/world-report-2015/en/

[3] Heinrich S, Rapp L, Rissmann U, Becker C, König HH. Cost of falls in old age: A systematic review. Osteoporosis International. 2009; 7(4):891-902. [DOI:10.1007/s00198-009-1100-1]

[4] Cosio-Lima LM, Reynolds KL, Winter C, Paolone V, Jones MT. Effects of physioball and conventional floor exercises on early phase adaptations in back and abdominal core stability and balance in women. Journal of Strength and Conditioning Research 2003; 17(4):721-5. [DOI:10.1519/00124278-200311000-00016]

[5] Avelar IS, Soares V, Barbosa RC, Andrade SR, Silva MS, Vieira MF. The influence of a protocol of aquatic exercises in postural control of obese elderly. Revista Andaluza de Medicina del Deporte. 2016; 9(4):2-7. [DOI:10.1016/j.ramd.2016.01.003]

[6] Hobeika CP. Equilibrium and balance in the elderly. Ear, Nose \& Throat Journal. 1999; 78(8):558-66. [DOI:10.1177/014 556139907800810]

[7] Bouisset S, Zattara M. Biomechanical study of the programming of anticipatory postural adjustments associated with voluntary movement. Journal of Biomechanics. 1987; 20(8):735-42. [DOI:10.1016/0021-9290(87)90052-2]

[8] Santos MJ, Kanekar N, Aruin AS. The role of anticipatory postural adjustments in compensatory control of posture: 2. Biomechanical analysis. Journal of Electromyography and Kinesiology. 2010; 20(3):398-405. [DOI:10.1016/j.jelekin.2010.01.002]

[9] Jagdhane S, Kanekar N, S Aruin AS. The effect of a four-week balance training program on anticipatory postural adjustments in older adults: A pilot feasibility study. Current Aging Science. 2016; 9(4):295-300. [DOI:10.2174/1874609809666160413113443]

[10] Kanekar N, Aruin AS. The effect of aging on anticipatory postural control. Experimental Brain Research. 2014; 232(4):1127-36. [DOI:10.1007/s00221-014-3822-3]

[11] Latash ML, Aruin AS, Neyman I, Nicholas JJ. Anticipatory postural adjustments during self inflicted and predictable perturbations in Parkinson's disease. Journal of Neurology, Neurosurgery, and Psychiatry. 1995; 58(3):326-34. [DOI:10.1136/jnnp.58.3.326] [PMID] [PMCID]

[12] Aruin AS, Kanekar N, Lee YJ. Anticipatory and compensatory postural adjustments in individuals with multiple sclerosis in response to external perturbations. Neuroscience Letters. 2015; 591:182-6. [DOI:10.1016/j.neulet.2015.02.050]

[13] Conceição JS, Schaefer de Araújo FG, Santos GM, Keighley J, dos Santos MJ. Changes in postural control after a ballkicking balance exercise in individuals with chronic ankle instability. Journal of Athletic Training. 2016; 51(6):480-90. [DOI:10.4085/1062-6050-51.8.02]

[14] Aruin AS, Kanekar N, Lee Y-J, Ganesan M. Enhancement of anticipatory postural adjustments in older adults as a result of a single session of ball throwing exercise. Experimental Brain Research. 2015; 233(2):649-55. [DOI:10.1007/s00221-014-4144-1]
[15] Siggeirsdottir K, Jonsson BY, Jonsson Jr H, Iwarsson S. The timed 'Up \& Go' is dependent on chair type. Clinical Rehabilitation. 2002; 16(6):609-16. [DOI:10.1191/0269215502cr529oa]

[16] Felicia AA, Paul G, Hausdorff JM. Risk factors for falls among older adults: A review of the literature. Maturitas. 2013; 75(1):51-61. [DOI:10.1016/j.maturitas.2013.02.009]

[17] Tinetti M, Speechley M, Ginter S. Risk factors for falls among elderly persons living in the community. New England Journal of Medicine. 1988; 319(26):1701-7. [DOI:10.1056/ NEJM198812293192604]

[18] Deandrea S, Lucenteforte E, Bravi F, Foschi R, La Vecchia C, Negri E. Risk Factors for Falls in Community-dwelling Older People: "A Systematic Review and Meta-analysis". Epidemiology. 2010; 21(5):658-68. [DOI:10.1097/EDE.0b013e3181e89905]

[19] Kanekar N, Aruin AS. Improvement of anticipatory postural adjustments for balance control: effect of a single training session. Journal of Electromyography and Kinesiology. 2015; 25(2):400-5. [DOI:10.1016/j.jelekin.2014.11.002]

[20] Inglin B, Woollacott M. Age-related changes in anticipatory postural adjustments associated with arm movements. Journal of Gerontology. 1988; 43(4):105-13. [DOI:10.1093/geronj/43.4.M105]

[21] Kanekar N, Aruin AS. Aging and balance control in response to external perturbations: Role of anticipatory and compensatory postural mechanisms. Age. 2014; 36(3):9621. [DOI:10.1007/ s11357-014-9621-8]

[22] Tsao H, Hodges PW. Persistence of improvements in postural strategies following motor control training in people with recurrent low back pain. Journal of Electromyography and Kinesiology. 2008; 18(4):559-67. [DOI:10.1016/j.jelekin.2006.10.012]

[23] Barry E, Galvin R, Keogh C, Horgan F, Fahey T. Is the timed up and go test a useful predictor of risk of falls in community dwelling older adults: A systematic review and meta-analysis. BMC Geriatrics. 2014; 14(1):1-14. [DOI:10.1186/1471-2318-14-14] 